\title{
Two or more intra AV nodal pathways in association with either a James or Kent extranodal bypass in 3 patients with paroxysmal supraventricular tachycardia ${ }^{1}$
}

\author{
Roworth A. J. Spurrell, Dennis Krikler, and Edgar Sowton \\ From the Departments of Cardiology, Guy's Hospital, and the Prince of Wales's Hospital, London
}

In 3 patients studied using His bundle electrograms and programmed intracardiac stimulation, evidence has been obtained of the functioning of multiple $A V$ nodal pathways in antegrade and retrograde directions during paroxysmal tachycardia due to an $A V$ nodal reciprocating mechanism. In 2 patients extranodal bypasses as seen in the pre-excitation syndromes were shown to be present in addition to the intra $A V$ nodal pathways. However, in one patient this extranodal bypass was not used in tachycardia and in the other it was concealed, only being brought to light when the tachycardia was terminated with intravenous verapamil. The significance of these findings is discussed.

A re-entry mechanism has long been postulated as the basis for certain types of abnormal cardiac rhythm; indeed, in I913 Mines first coined the term 'reciprocating rhythm'. Since then extensive animal experimental work (Moe and Mendez, 1966; Mendez and Moe, 1966; Watanabe and Dreifus, 1965) has shown the presence of two functionally separate pathways within the atrioventricular node, one conventionally named alpha, with a short refractory period and a long conduction time, the other the beta pathway, with a long refractory period and a short conduction time. If a premature impulse is conducted antegradely down the alpha pathway when the beta pathway is refractory, it may be able to return retrogradely up the beta pathway, and produce an atrial reciprocal beat. A similar sequence can be initiated in the reverse direction by a ventricular premature beat. In I97I, Janse et al. showed that such a circus movement within the AV node was the basis for a supraventricular tachycardia in an isolated rabbit atrioventricular node preparation. A reciprocal mechanism as a basis for supraventricular tachycardia in man was suggested as early as 1923 by Iliescu and Sebastiani, and by Drury in r 924 . More recently, intracardiac recording combined with programmed electrical stimulation has sup-

Received 2I July 1972.

${ }^{1}$ Part of this work was supported by a grant from the British Heart Foundation. ported the belief that a reciprocal mechanism within the AV node forms the basis for many cases of supraventricular tachycardia in man (Durrer, Schuilenburg, and Wellens, 1969; Bigger and Goldreyer, 1970; Wellens, 1971a). However, the anatomical structure of the AV node (James, 196I) suggests that multiple pathways are conceivable.

We now present the findings in 3 patients with AV nodal reciprocal tachycardias in whom there is evidence that more than two AV pathways may be implicated.

\section{Patients and methods}

Three patients were studied: one female and two male. The details of pertinent clinical and electrocardiographic findings are shown in Table I. Each patient gave a threeyear history of rapid heart action; Cases $I$ and 3 were thought also to have ischaemic heart disease.

His bundle electrograms using the technique described by Scherlag et al. (1969) were recorded in all 3 patients, at which time all treatment had been stopped for 7 days. Medications previously administered are cited in Table I. Four bipolar catheters were introduced into the femoral veins by direct puncture and were positioned as follows: (a) one catheter high in the right atrium for recording a high atrial electrogram; (b) one catheter low in the right atrium for recording a low atrial electrogram; (c) one catheter across the tricuspid valve for recording a His bundle electrogram; and (d) one catheter positioned in the right atrium or right ventricle for atrial 
or ventricular pacing, or for introducing atrial or ventricular premature beats.

The recordings were made with an Elema Mingograf $8 \mathrm{I}$ at a paper speed of $100 \mathrm{~mm} / \mathrm{sec}$. The His bundle electrogram was recorded with the frequency response set at 45-500 cycles/sec. A surface electrocardiogram was recorded simultaneously.
Drugs taken before Electrocardiogram

investigation

Using a Devices $4270^{1}$ stimulator the heart was driven by atrial pacing at a rate of 100 beats a minute and a test stimulus, of $2 \mathrm{msec}$ pulse width, was introduced every sixth beat into the atrium, with a known delay following the preceding basic driving stimulus. This delay was gradually reduced until a tachycardia was 1 Devices Ltd., Welwyn Garden City, Herts.

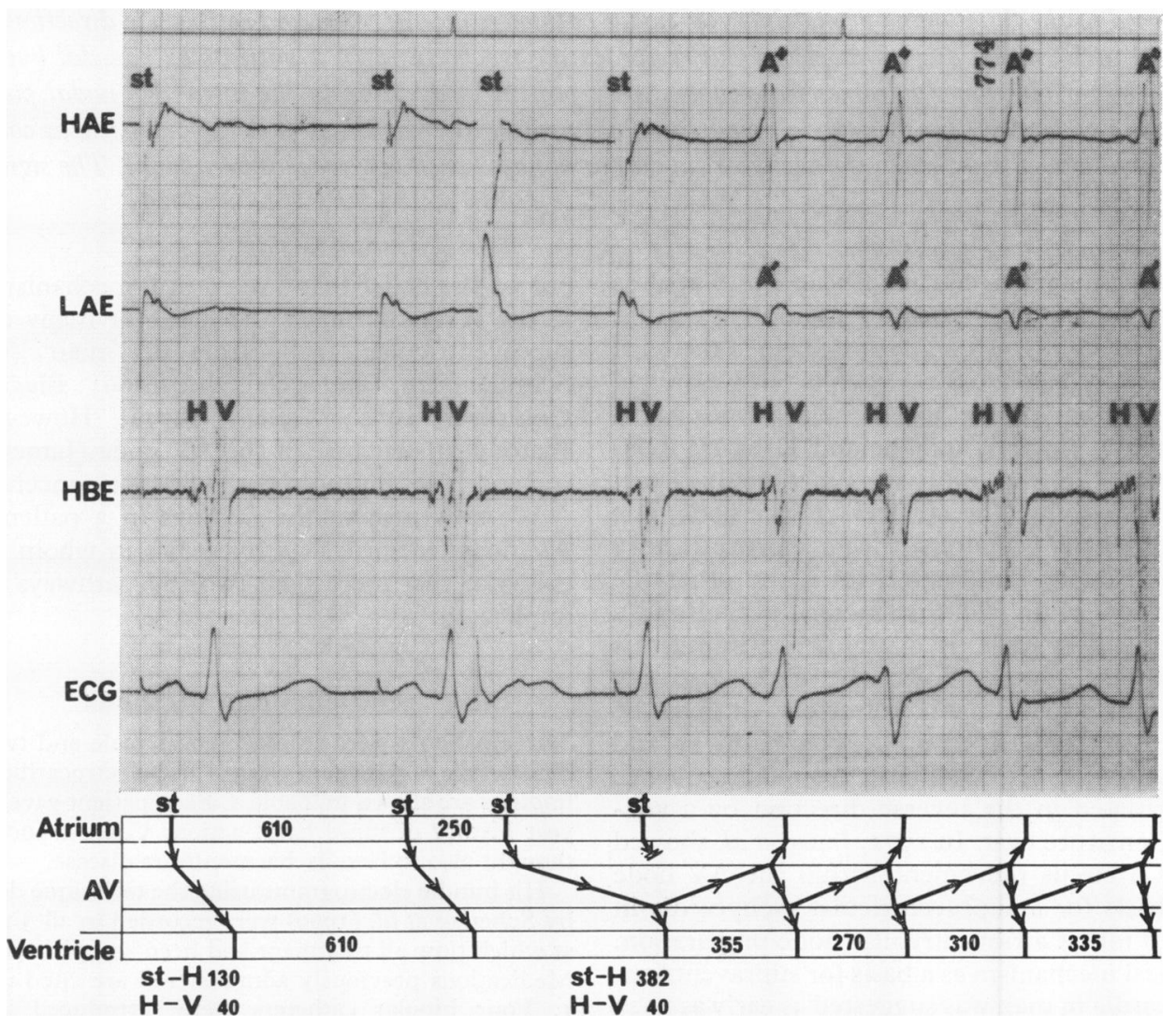

FIG. I Case I. Initiation of tachycardia by one atrial premature beat. The first two beats are atrially paced beats and the third beat is an induced atrial premature beat which starts the tachycardia. $H A E=$ high atrial electrogram; $L A E=$ low atrial electrogram; $H B E=H i$ bundle electrogram; $E C G=$ surface electrocardiogram; $A V=$ atrioventricular node; st $=$ stimulus; $H=H$ is bundle depolarization; $V=$ ventricular depolarization; $A^{\prime}=$ atrial depolarization on the low atrial electrogram; $A^{+}=$atrial depolarization on the high atrial electrogram. 
started by an atrial premature beat which had a long AV nodal conducting time. The influence of atrial and ventricular premature beats introduced during the tachycardia was studied. The study was completed by giving $10 \mathrm{mg}$ verapamil by intravenous injection; in all three patients this terminated the tachycardia (Schamroth, Krikler, and Garrett, 1972).

\section{Results}

\section{Case I}

The tachycardia could be consistently started by a single atrial premature beat with a delay of 250 msec from the preceding atrially paced beat (Fig. I). During tachycardia each QRS complex was identical with those seen in sinus rhythm and was pre- ceded by a His depolarization, indicating that the tachycardia was supraventricular in origin. That there was retrograde conduction to the atria in tachycardia is indicated by the fact that atrial depolarization was later in the high than in the low atrial electrogram. Single atrial or ventricular premature beats introduced during the tachycardia were followed by pauses that were less than compensatory. During the tachycardia (Fig. 2) there are regularly alternating cycles, respectively $285 \mathrm{msec}$ and $340 \mathrm{msec}$ in duration. This is due to alternation of the antegrade conduction times (indicated by the $A^{\prime}-H$ intervals); retrograde conduction (indicated by the $\mathrm{H}-\mathrm{A}^{\prime}$ intervals) was $50 \mathrm{msec}$ and the same for each cycle. These findings suggest the
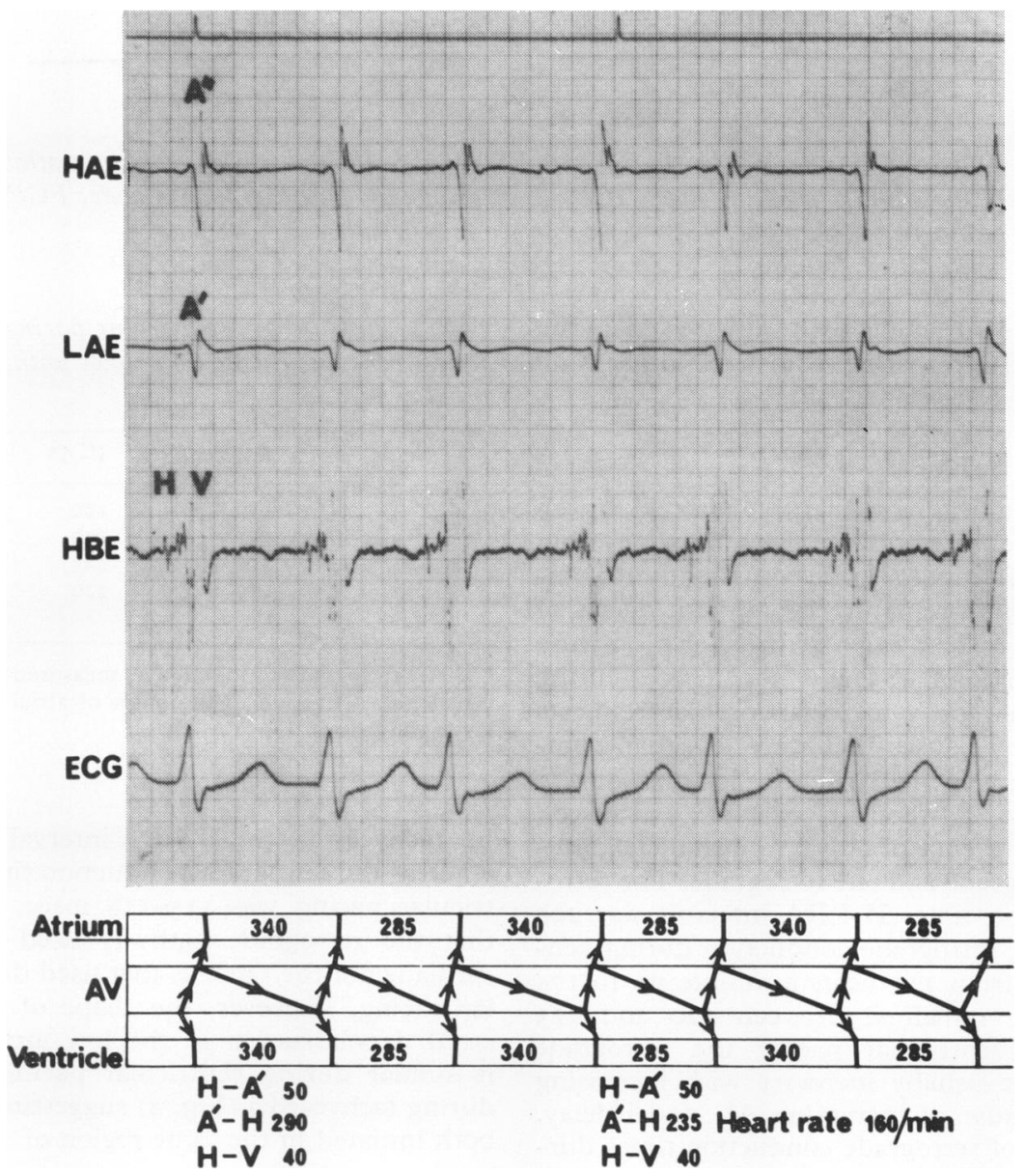

FIG. 2 Case I. Recording obtained during tachycardia showing alternation of atrial and ventricular cycle lengths. This is due to alternation of antegrade conduction, retrograde conduction times being the same for both the long and short cycles. 
Atrium

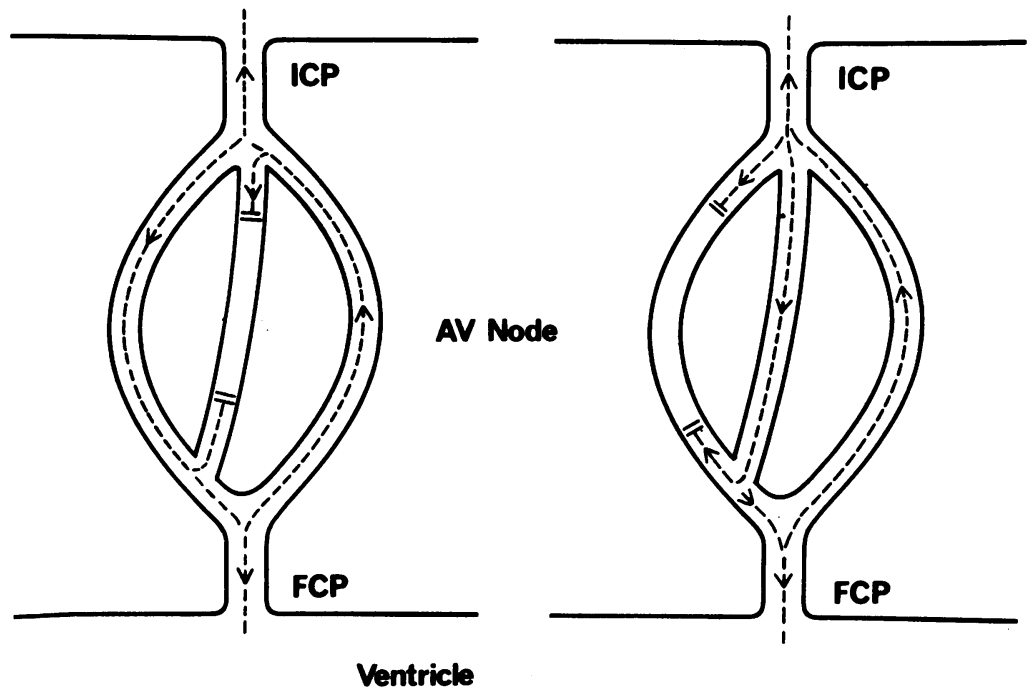

FI G. 3 Diagram illustrating the three intra AV nodal pathways in Case I. Two pathways are used antegradely and one retrogradely. ICP = initial common pathway in $A V$ node; $F C P=$ final common pathway in $A V$ node.

presence of three intranodal pathways, two operating antegradely and alternating with each other, and one functioning retrogradely (Fig. 3 ).

\section{Case 2}

The tachycardia was consistently initiated by an atrial premature beat with a delay ranging from 220-400 msec. It could also be consistently terminated by two atrial premature beats, with delays of I 95 and $335 \mathrm{msec}$, or by two ventricular premature beats, with delays of 185 and 390 msec. During tachycardia atrial or ventricular premature beats were followed by pauses that were less than compensatory. The ventricular cycle lengths during tachycardia (Fig. 4) were all constant (310 msec), indicating the presence of only two intranodal pathways - one antegrade and one retrograde. The retrograde conduction time (H-LRA interval) was 200 msec. However, retrograde conduction during ventricular pacing from I IO beats a minute up to 175 beats a minute remained between 120 and 123 msec. During ventricular pacing the retrograde conduction time usually increases with increasing heart rates because of increasing AV nodal delay. The constancy of retrograde conduction times during ventricular pacing in this patient (Table 2) suggests the presence of a tract bypassing the atrioventricular node, e.g. the posterior internodal tract (James, 1963). The retrograde conduction time dur-
TABLE 2 Case 2: Ventricular pacing results showing almost constant conduction times with increasing ventricular rate

\begin{tabular}{lll}
\hline Rate/min & $V-A^{\star}$ \\
\hline 110 & 120 \\
130 & 120 \\
150 & 123 \\
175 & 120
\end{tabular}

$\star \mathrm{V}-\mathrm{A}=$ retrograde conduction as measured from the onset of ventricular activation to the onset of atrial depolarization in low right atrium.

ing tachycardia (the H-LRA interval) was $200 \mathrm{msec}$ whereas the retrograde conduction time during ventricular pacing was II5-1I8 msec. This suggests that the retrograde pathway used during tachycardia is not the same as that used during ventricular pacing. However, the shape of the retrograde atrial depolarization in the low atrial electrogram is similar during ventricular pacing (Fig. 5) and during tachycardia (Fig. 4) suggesting that they are both initiated in the same region of the atria.

\section{Case 3}

The tachycardia was consistently initiated by an atrial premature beat occurring with a delay of 300 

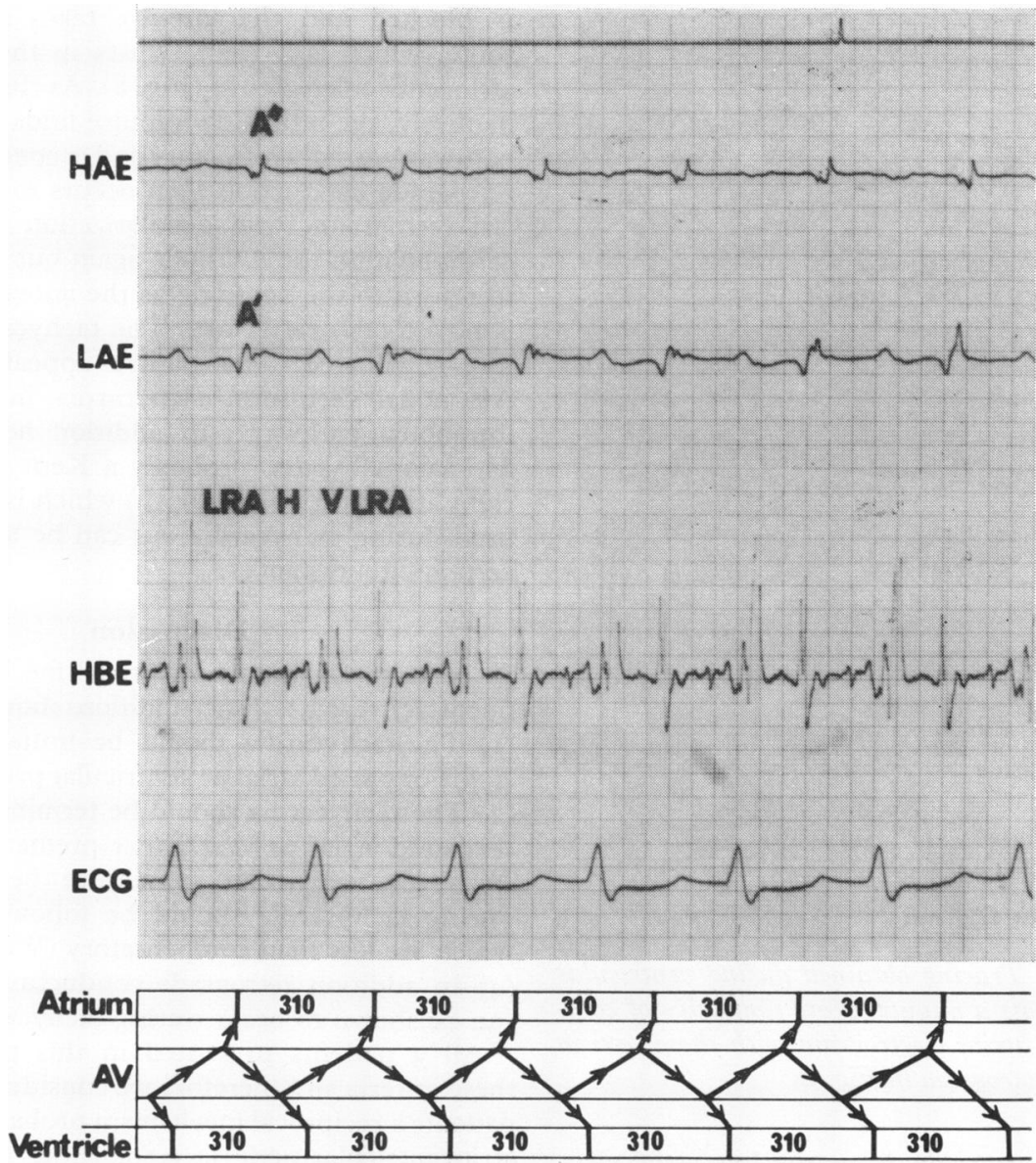

H-LRA 200

LRA-H 110 Heart rate 190/min

H-V 55

FIG. 4 Case 2. Recording obtained during tachycardia showing constant atrial and ventricular cycle lengths. $L R A=$ low right atrial depolarization as recorded on the His bundle electrogram.

msec following the preceding atrially-driven beat. The tachycardia could not be stopped by an atrial premature beat but was consistently terminated by a ventricular premature beat with a delay of 210 msec following the preceding beat. Ventricular premature beats produced during the tachycardia were always followed by a pause that was less than compensatory. During tachycardia it can be seen that the $R R$ intervals alternate, one being $310 \mathrm{msec}$ and the other $420 \mathrm{msec}$ (Fig. 6). This is clearly due to alternation of conduction times in the antegrade pathway (as measured by the LRA-H interval): this was alternately IIO msec and $220 \mathrm{msec}$. On the other hand, the retrograde pathway (as measured by the H-LRA interval) has a conduction time of
$200 \mathrm{msec}$ and is the same for each cycle. There must therefore be three functionally separate pathways: two antegrade, alternating with each other; and one retrograde.

When the tachycardia was finally terminated using intravenous verapamil a fourth pathway became manifest. Fig. 7 illustrates the last four beats of the tachycardia and the first two beats of sinus rhythm. The last QRS complex of the tracing is seen to have a delta wave following the $P$ wave, and the His spike on the His bundle electrogram occurs simultaneously with the onset of the delta wave on the external electrocardiogram. Delta waves were seen on several occasions during the study but had never been seen on resting electrocardiograms in the past. In addi- 
tion, the third retrograde $A^{\prime}$ on the low atrial electrogram occurred prematurely, at $308 \mathrm{msec}$ instead of $370 \mathrm{msec}$. It also has a different contour from the other retrograde $A^{\prime}$ depolarizations in that it has greater positivity; it is very similar to that of atrial depolarization occurring in the low atrial electrogram from the first sinus beat. This difference in contour may be explained using the diagram to the right of the tracings; this shows the position of the catheter used for recording the low atrial electrogram. Both sinus impulses and impulses arriving from the region of the right lateral atrioventricular groove will give predominantly positive deflections as recorded by this catheter. The presence of a delta wave and a premature retrograde atrial depolarization with this contour provides strong evidence of an anomalous Kent bypass in the right lateral atrioventricular groove as occurs in the Wolff-Parkinson-White syndrome type B.

The termination of the arrhythmia by verapamil is probably due to an increase in AV nodal conduction time, so that, following the second retrograde atrial depolarization $\left(A^{\prime}\right)$, the retrograde pathway

is blocked and the impulse takes an alternative route, which is a Kent bypass in the right lateral atrioventricular groove (Fig. 8). As this arrived prematurely, the returning impulse finds the antegrade pathway partially refractory and is conducted slowly, retrograde conduction then occurs to the atria (the last retrograde atrial depolarization of the tachycardia) within the AV node again but is now unable to return to the ventricle as the antegrade pathway is now totally refractory. The tachycardia is therefore terminated. This patient appears to have an AV nodal reciprocal tachycardia involving three intranodal pathways; in addition he also has an anomalous bypass (probably a Kent bundle in the right atrioventricular groove) which is not normally used during tachycardia but can be exposed under certain circumstances.

\section{Discussion}

In order to diagnose a reciprocal mechanism in AV nodal tachycardias, four conditions should be fulfilled. (I) The tachycardia should be initiated by either single or paired atrial or ventricular premature beats. (2) The tachycardia should be terminated by single or paired atrial or ventricular premature beats. (3) Atrial or ventricular premature beats occurring during tachycardia should be followed by pauses which are less than compensatory (Wellens, I97Ib). (4) In addition retrograde conduction to the atria can be shown to occur during tachycardia.

All 3 patients presented in this paper fulfilled these criteria and therefore we consider that in these patients a reciprocal mechanism probably within the atrioventricular node is responsible for the tachycardia.

During AV nodal reciprocal tachycardia there is functional dissociation within the AV node into two pathways, one conducting antegradely and one conducting retrogradely. This characteristically leads to a tachycardia with a given cycle length which tends to be constant. However, alternating cycle lengths during supraventricular tachycardia have been reported (Barker, Johnston, and Wilson, I943; Langendorf, 1958) and have more recently been thought to be due to alternating functional pathways within the atrioventricular node (Moe, Childers, and Merideth, 1968). Case I has an alternating antegrade pathway, but the same retrograde pathway is used, as illustrated in Fig. 3. The possibility of numerous different pathways within the atrioventricular node is not surprising when one considers the histology of the upper part of the AV node (James, I96I). There is a complex network of cells in the upper part of the AV node which only becomes finally organized into parallel pathways in the lower part of the node. Hence an impulse arriving 


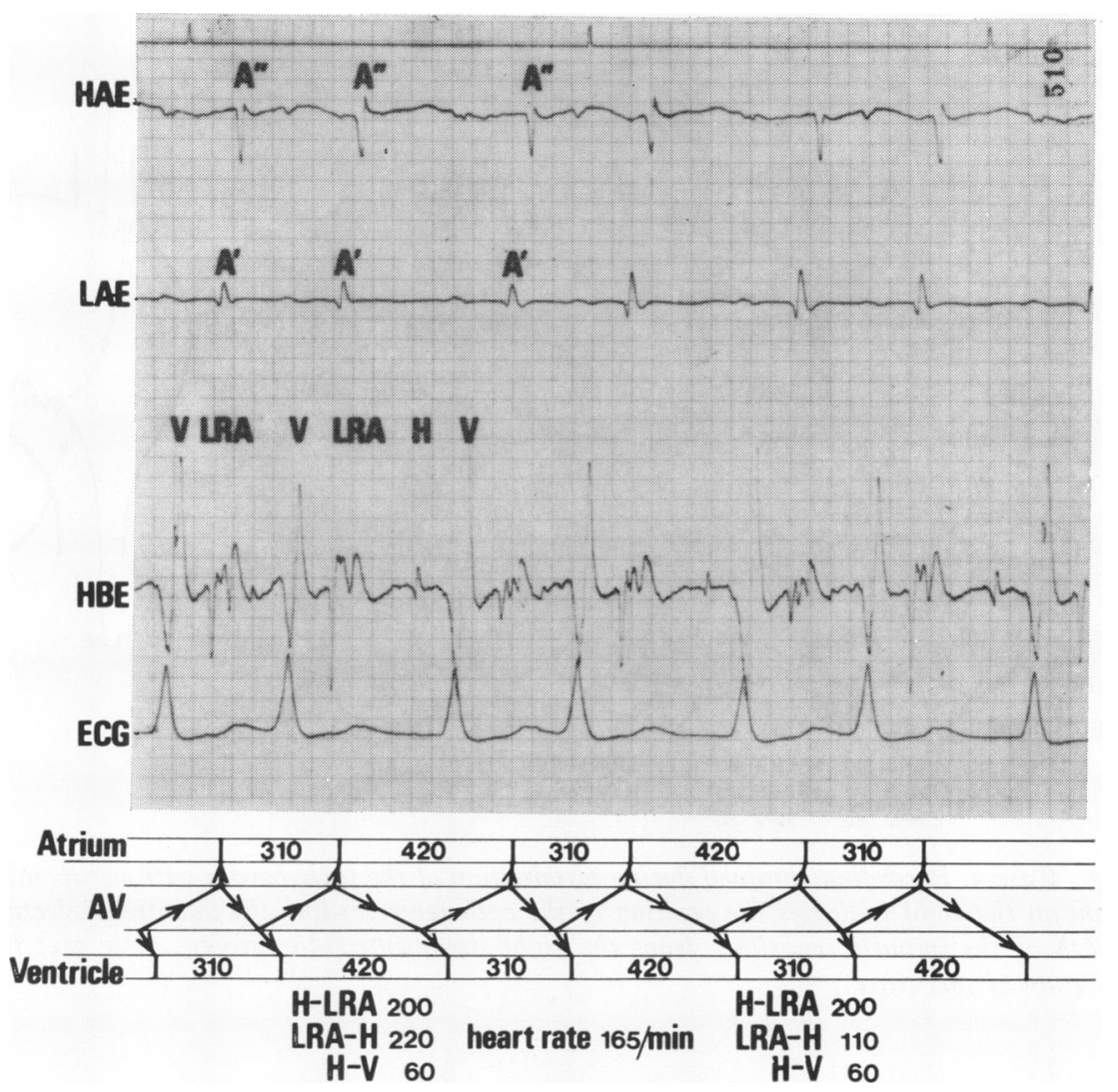

FIG. 6 Case 3. Recording obtained during tachycardia. Note the alternation of atrial and ventricular cycle lengths. This is seen to be due to alternation in antegrade conduction, retrograde conduction times being the same for both the long and the short cycles.

in the upper part is faced with numerous pathways of varying lengths and electrophysiological properties which it has to traverse before reaching the more orderly structure of the lower part of the AV node. It is perhaps not surprising that under certain circumstances different pathways in the upper part of the node are preferentially taken. The presence of cross-connexions within the upper part of the node may allow an antegrade impulse to cross to another pathway and return retrogradely to the atrium.

In Case I the retrograde conduction time is short ( $50 \mathrm{msec}$ ), and it is possible that retrograde conduction in this patient is via a James AV nodal bypass and not an intranodal pathway. However, no further support for this view was obtained from the surface electrocardiogram or from atrial or ventricular pacing studies.

Patients with a PR interval of less than $0.12 \mathrm{sec}$, no delta wave, and a normal QRS who get paroxysmal tachycardia are thought to have the LownGanong-Levine syndrome (Lown, Ganong, and Levine, 1952) in which there is a James bypass of the atrioventricular node. The mechanism of the tachycardia in these patients is thought to be reciprocal in that an impulse passes antegradely in the AV node and then retrogradely via the James bypass to re-excite the atria; there is therefore only one functional pathway within the AV node in this situation. Case 2 has evidence of a James bypass of the AV node as indicated by the short PR interval on the resting electrocardiogram and constant but short ventriculoatrial conduction times during ventricular pacing up to 180 a minute (Castellanos et al., I97I). However, during tachycardia this patient does not appear to use this bypass as indicated by the longer retrograde conduction times during tachycardia at a similar heart rate to the maximum 


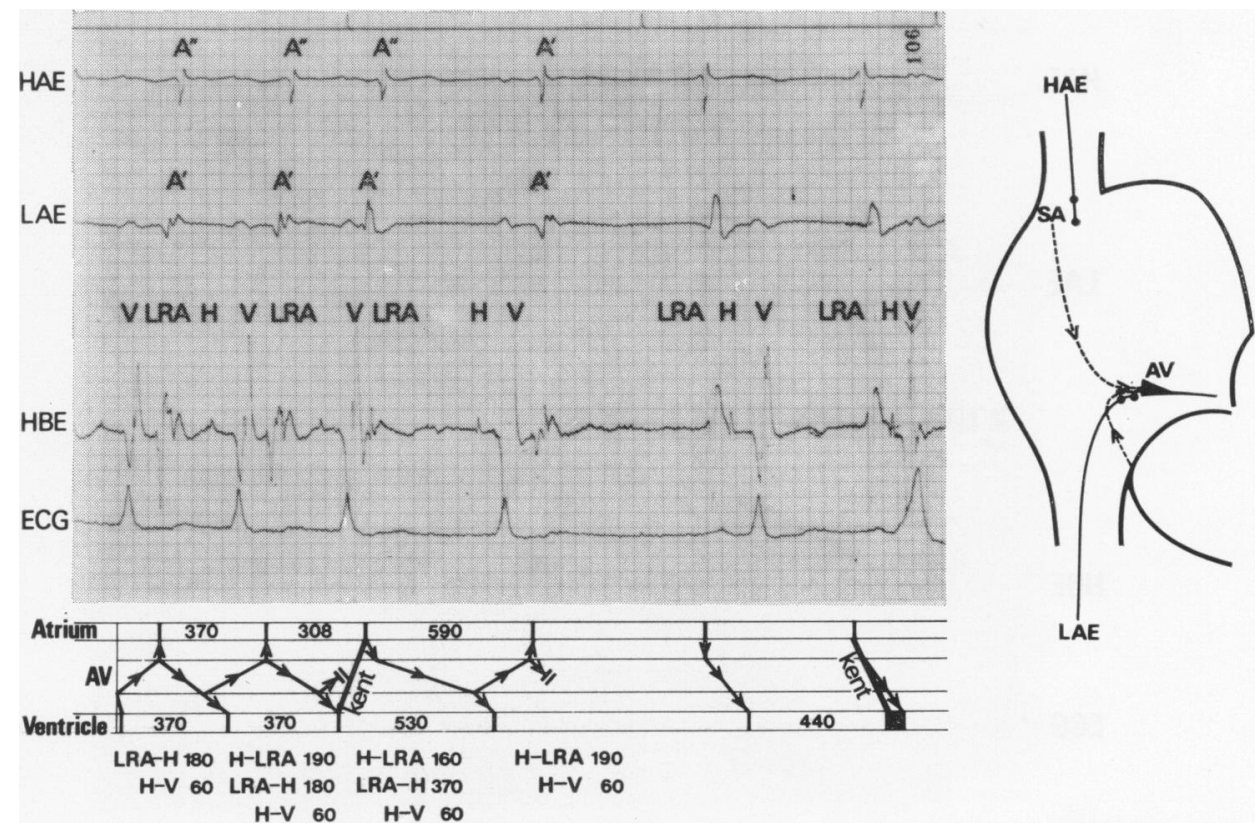

FIG. 7 Case 3. Recording obtained during termination of the tachycardia with verapamil. The diagram on the right indicates the position of the catheter recording the low atrial electrogram and path of the impulse travelling from the right atrioventricular groove. (See text for full explanation of this trace.)

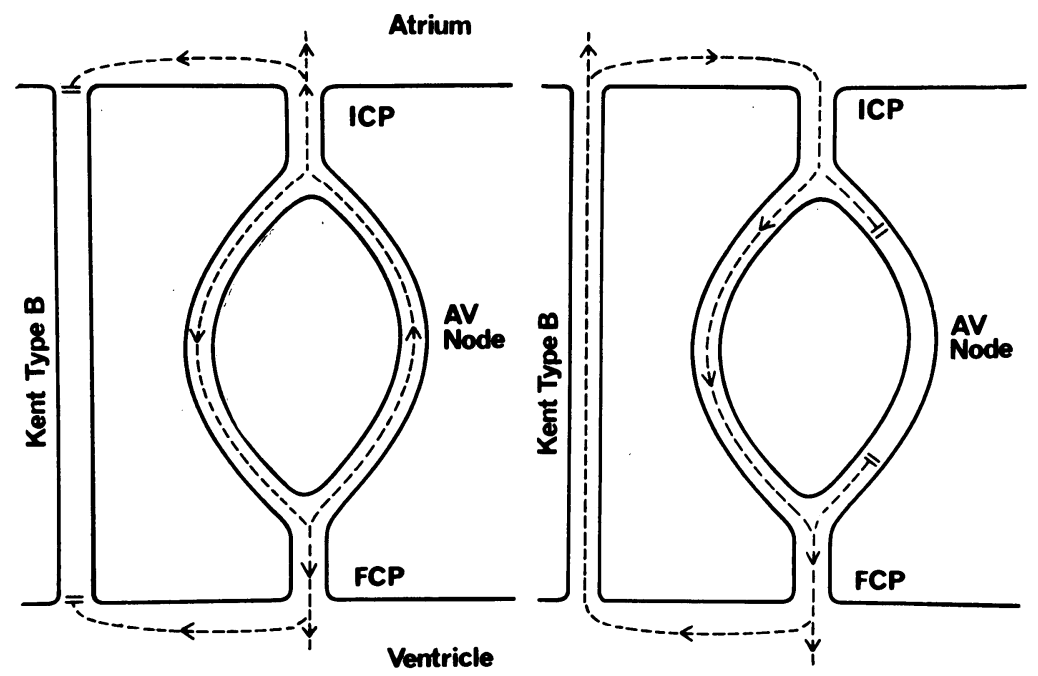

FIG. 8 Case 3. Diagram illustrating on the left a reciprocating tachycardia within the atrioventricular node, with the impulse blocked in the Kent bundle. On the right, during termination of the tachycardia the impulse is blocked retrogradely in the AV node but now passes retrogradely to the atrium via the Kent bundle. 
rate during ventricular pacing. These times are measured from the His potential to the low atrial potential during tachycardia, whereas during ventricular pacing the retrograde conduction is measured from the onset of ventricular activation to the time of arrival of the impulse in the low right atrium, a factor that should further exaggerate the difference in retrograde conduction times during tachycardia and ventricular pacing. It seems reasonable therefore to postulate a different retrograde pathway during tachycardia from the James bypass that appears to be used during ventricular pacing. This retrograde pathway is likely to be within the atrioventricular node and arrives in the atrium at a similar site as the insertion of the James bypass as indicated by similar vectors of the atrial potentials in the low atrial electrogram, both during ventricular pacing and during tachycardia. This is not unexpected as the James bypass is thought to be part of the posterior internodal tract which connects the sinoatrial node with both the upper and lower parts of the atrioventricular node (James, 1963). Case 2 therefore has evidence of the Lown-Ganong-Levine syndrome, but when in tachycardia he did not use the James bypass in the reciprocal mechanism, two intranodal pathways being concerned.

The third patient fulfilled the criteria for a reciprocal tachycardia and during the tachycardia the RR intervals alternated in duration indicating the presence of three pathways. The two antegrade pathways alternated in length, but the same retrograde pathway is used during each cycle (Fig. 6). After verapamil was given, the tachycardia was terminated, and a delta wave for the first time became manifest on the surface electrocardiogram. Just before the tachycardia was terminated retrograde conduction appeared to occur prematurely in one beat via a Kent bundle situated in the right lateral atrioventricular groove. As this was the only occasion during the tachycardia that this occurred, the Kent bundle was presumably used only because the usual retrograde pathway within the AV node had been blocked by verapamil. This patient therefore exhibited three intranodal AV pathways, two antegrade and one retrograde, and there was also evidence of an anomalous Kent bypass only used during tachycardia under the special circumstances described. This is somewhat different from the case described by Friedberg and Schamroth (1972), a similar patient who had two antegrade intranodal pathways but who used a Kent bypass consistently for retrograde conduction during tachycardia. When a patient with clear electrocardiographic evidence of the Wolff-Parkinson-White syndrome has reciprocating tachycardia, it is logical to link the arrhythmias with the presence of the anomalous pathway.
Our experience with Case 2, who also had a preexcitation syndrome, shows that this may not always be valid. Indeed, Coumel and Waynberger (1972) studied a patient with the Wolff-Parkinson-White syndrome type $B$ in whom AV nodal reciprocating tachycardia was demonstrated which did not involve the anomalous pathway. As surgical section of the anomalous pathway is sometimes considered in the treatment of resistant tachycardias in the WolffParkinson-White syndrome, the precise mechanism of the arrhythmia must first be shown and the role of the extranodal bypass carefully assessed. Further examples of presentations of tachycardias using multiple intranodal and/or extranodal pathways will be found only by careful scrutiny of recordings. The knowledge that more than one anomalous tract may be found in the Wolff-Parkinson-White and related syndromes (Lev, Gibson, and Miller, 1955; Coumel et al., I97 ; Ramachandran, 1972) is highly relevant in this context.

In Case 3, the anomalous pathway seems incidental to the presentation. However, there are circumstances where it may be virtually impossible to demonstrate the presence of an anomalous pathway displaying antegrade conduction, during sinus rhythm, in the Wolff-Parkinson-White syndrome (De la Fuente, Sasyniuk, and Moe, I97I), though dangerous arrhythmias may nevertheless occur (Yahini, Zahavi, and Neufeld, 1964; Krikler, 1972). The possible provocation of anomalous conduction by verapamil deserves further evaluation: whether this would be entirely by the blocking of intranodal conduction; or whether there is enhancement of conduction in the anomalous pathway, is under investigation.

\section{Conclusion}

The finding in 3 patients of evidence of the functioning of multiple AV nodal pathways, in antegrade and retrograde directions, during paroxysmal tachycardia due to an AV nodal reciprocating mechanism, is in keeping with the anatomical and physiological characteristics of the AV node. The studies also showed, in two cases, the presence of extranodal bypasses as seen in pre-excitation syndromes, but in one patient this bypass was not used, and in the other it was concealed, only being brought to light when the tachycardia was terminated by intravenous verapamil. Thus in not all patients with paroxysmal tachycardias complicating pre-excitation is the extranodal bypass consistently used in the reciprocal mechanism that produces the arrhythmia. Detailed analysis of patients with AV nodal tachycardias may provide more information about the behaviour of various functional pathways within the AV node. 


\section{References}

Barker, P. S., Johnston, F. D., and Wilson, F. N. (1943). Auricular paroxysmal tachycardia with alternation of cycle length. American Heart fournal, 25, 799.

Bigger, J. T., and Goldreyer, B. N. (1970). The mechanism of supraventricular tachycardia. Circulation, 42, 673.

Castellanos, A., Castillo, C. A., Agha, A. S., and Tessler, M. (197I). His bundle electrograms in patients with short P-R intervals, narrow QRS complexes, and paroxysmal tachycardias. Circulation, 43, 667.

Coumel, P., and Waynberger, M. (1972). Les tachycardies par rythme réciproque au cours du syndrome de WolffParkinson-White. Coeur et Médecine Interne, II, 77.

Coumel, P., Waynberger, M., Slama, R., and Bouvrain, Y. (I97I). Interêt de l'enregistrement des potentiels hisiens au coeurs du syndrome de Wolff-Parkinson-White. A propos de six observations. Acta Cardiologica, 26, 188.

De la Fuente, D., Sasyniuk, B., and Moe, G. K. (I971). Conduction through a narrow isthmus in isolated canine atrial tissue. A model of the W-P-W syndrome. Circulation, $44,803$.

Drury, A. N. (1924). Paroxysmal tachycardia of A-V nodal origin, exhibiting retrograde heart-block and reciprocal rhythm. Heart, II, 405.

Durrer, D., Schuilenburg, R. M., and Wellens, H. J. J. (1969). The role of the atrio-ventricular junction in the genesis of arrhythmias in the human heart. Proceedings. Koninklijke Nederlandse Akademie van Wetenschappen te Amsterdam, 72, 50I.

Friedberg, H. D., and Schamroth, L. (1972). Three atrioventricular pathways: reciprocating tachycardia with alternation of conduction time. Fournal of Electrocardiology. In the press.

Iliescu, C. C., and Sebastiani, A. (1923). Notes on the effects of quinidine upon paroxysms of tachycardia. Heart, 10, 223.

James, T. N. (196I). Morphology of the human atrioventricular node with remarks pertinent to its electrophysiology. American Heart fournal, 62, 756.

James, T. N. (1963). The connecting pathways between the sinus node and $\mathrm{A}-\mathrm{V}$ node and between the right and the left atrium in the human heart. American Heart fournal, 66, 498.

Janse, M. J., Van Capelle, F. J. L., Freud, G. E., and Durrer, D. (197I). Circus movement within the A-V node as a basis for supraventricular tachycardia as shown by multiple microelectrode recording in the isolated rabbit heart. Circulation Research, 28, 403.
Krikler, D. M. (1972). The Wolff-Parkinson-White and related syndromes. An electrocardiographic appraisal. M.D. Thesis, University of Cape Town.

Langendorf, R. (1958). Alternation of A-V conduction time. American Heart fournal, 55, I8I.

Lev, M., Gibson, S., and Miller, R. A. (1955). Ebstein's disease with Wolff-Parkinson-White syndrome. Report of a case with histopathalogic study of possible conduction pathways. American Heart fournal, 49, 724.

Lown, B., Ganong, W. F., and Levine, S. A. (1952). The syndrome of short P-R interval, normal QRS complex and paroxysmal rapid heart action. Circulation, 5, 693.

Mendez, C., and Moe, G. K. (1966). Demonstration of a dual A-V nodal conduction system in the isolated rabbit heart. Circulation Research, 19, 378.

Mines, G. R. (1913). On dynamic equilibrium in cardiac muscle. Fournal of Physiology, 46. In Proceedings of the Physiological Society, p. xxiii.

Moe, G. K., Childers, R. W., and Merideth, J. (1968). An appraisal of 'supernormal' A-V conduction. Circulation, 38, 5 .

Moe, G. K., and Mendez, C. (1966). The physiologic basis of reciprocal rhythm. Progress in Cardiovascular Diseases, 8,461 .

Ramachandran, S. (1972). Wolff-Parkinson-White syndrome: conversion of type A to type B electrocardiographic changes. Circulation, 45, 529.

Schamroth, L., Krikler, D. M., and Garrett, C. (1972). Immediate effects of intravenous verapamil in cardiac arrhythmias. British Medical fournal, r, 660.

Scherlag, B. J., Lau, S. H., Helfant, R. H., Berkowitz, W. D., Stein, E., and Damato, A. N. (1969). Catheter technique for recording His bundle activity in man. Circulation, 39, 13.

Watanabe, Y., and Dreifus, L. S. (1965). Inhomogeneous conduction in the A-V node. American Heart fournal, 70, 505.

Wellens, H. J. J. (I971a). Electrical Stimulation of the Heart in the Study and Treatment of Tachycardias, p. 40. H. E. Stenfert Kroese N.V., Leiden.

Wellens, H. J. J. (197rb). Electrical Stimulation of the Heart in the Study and Treatment of Tachycardias, p. 14. H. E. Stenfert Kroese N.V., Leiden.

Yahini, J. H., Zahavi, I., and Neufeld, H. N. (1964). Paroxysmal atrial fibrillation in Wolff-Parkinson-White syndrome simulating ventricular tachycardia. American fournal of Cardiology, 14, 248.

Requests for reprints to Dr. R. A. J. Spurrell, Department of Cardiology, Guy's Hospital, London SEI 9RT. 